\title{
Alterações Cognitivas, Comunicativas e Emocionais APÓs Lesão HeMisfÉrica Direita: EM Busca de uma Caracterização da SíndRome do HeMisfÉrio Direito ${ }^{1}$
}

\author{
Rochele Paz Fonseca ${ }^{2}$, Gabriela Damasceno Ferreira ${ }^{3}$, Francéia Veiga \\ Liedtke $^{3}$, Juliana de Lima Müller ${ }^{3}$, Thaís Ferrugem Sarmento ${ }^{3}$ e Maria \\ Alice de Mattos Pimenta Parente ${ }^{4}$ \\ Universidade Federal do Rio Grande do Sul - UFRGS
}

O conjunto de sinais e sintomas observados após um acometimento neurológico no hemisfério direito pode ser denominado de Síndrome do Hemisfério Direito-SHD. Esse ensaio teórico tem por objetivo apresentar uma caracterização desse quadro neuropsicológico. A SHD é, então, caracterizada por déficits nas funções cognitivas atenção, percepção, memória, praxias e funções executivas, com a presença de anosognosia, heminegligência sensorial, prosopagnosia, alterações de memória visuoespacial e de trabalho, dispraxia construtiva e disfunção executiva. Quanto às habilidades comunicativas, a SHD engloba alterações nos componentes discursivo, pragmático-inferencial, léxico-semântico e prosódico. Os déficits de processamento emocional incluem dificuldades de compreensão e produção de emoções a partir de expressões faciais ou emissões vocais e alterações neuropsiquiátricas. Há, no entanto, uma heterogeneidade na sua manifestação. Tendo em vista essa variabilidade de sinais e sintomas, mais estudos de caso e de grupo com indivíduos lesados de hemisfério direito devem ser conduzidos para um melhor entendimento da SHD.

Descritores: Neuropsicologia. Cognição. Comunicação. Emoção. Hemisfério direito.

1 Artigo originado da tese de doutoramento da primeira autora defendida em 2006 no Departamento de Psicologia - UFRGS. Apoio financeiro da CAPES, PET e CNPq.

2 Pesquisadora do Laboratório de Neuropsicolingüistica do Instituto de Psicologia - UFRGS. Endereço eletrônico: rochele.fonseca@gmail.com

3 Graduandas de Psicologia - UFRGS.

4 Docente do Instituto de Psicologia - UFRGS. 
$\mathrm{O}$ interesse pela dicotomia hemisfério esquerdo (HE) versus hemisfério direito (HD) tem sido bastante evidenciado na literatura atual. Essa preocupação está presente desde o surgimento da Neuropsicologia, em 1861.

Tal dicotomia diz respeito à noção de dominância cerebral, cujo histórico se confunde com o nascimento e o desenvolvimento da própria neuropsicologia. Isso porque o estudo anátomo-clínico de Paul Broca que intitulou o HE como dominante para a linguagem é considerado o marco inicial da Neuropsicologia (Burns, Halper, \& Mogil, 1985). Conforme Brookshire (2003), o conceito de dominância cerebral está baseado na noção de que o HE é responsável pelo processamento da função linguagem, sendo considerado o hemisfério dominante, maior e ativo. Em contrapartida, o HD passa a ser considerado o dominado, o menor e o passivo. Outro fator que contribuiu para que o $\mathrm{HE}$ fosse considerado o hemisfério dominante foi o seu papel essencial também no movimento voluntário (Marchetti, Carey, \& Della Sala, 2005).

Neste curso histórico, as décadas de 1860 e 1870 foram marcadas pelos trabalhos de Broca, que demonstraram que alterações na expressão lingüística estavam associadas a lesões no lobo frontal esquerdo, em 1861, e os trabalhos de Wernicke que evidenciaram relações entre alterações na compreensão da linguagem e lesões no lobo temporal esquerdo em 1874. Em 1876, Jackson sugeriu um papel importante do HD no processamento da percepção visual (Bryan, 1995).

No entanto, apenas 100 anos após o HE ficar conhecido cientificamente como dominante, o entendimento sobre o HD começou a mudar. Depois das duas grandes Guerras Mundiais, dados com lesados de hemisfério direito (LHD) confirmaram a sugestão de Jackson. Juntamente com os estudos com indivíduos lesados pós-guerra, na década de 1940, foi desenvolvida a comissurectomia - split brain, técnica cirúrgica que possibilitava acesso independente às funções de cada hemisfério através da separação das metades direita e esquerda do cérebro pela seç̧ão do corpo caloso em pacientes com epilepsia.

Em relação às especializações hemisféricas das funções cognitivas, há atualmente um certo consenso na literatura. O HE é mais associado às habilidades de pensamento lingüístico, raciocínio analítico, memória verbal e produção e compreensão da linguagem. $\mathrm{O} \mathrm{HD}$, em contrapartida, é associado às seguintes funções cognitivas: atenção, percepção e memória visuo-espaciais, 
Alterações Cognitivas, Comunicativas e Emocionais após Lesão Hemisférica...

esquema corporal, inteligência social e emocional, reconhecimento de expressões faciais e habilidade musical (Myers, 2001).

Desse modo, nota-se que desde a década de 1960 o papel do HD na cognição, na comunicação e na emoção tem sido cada vez mais investigado pela ciência neuropsicológica, o que evidencia que esse hemisfério deixou de ser negligenciado. Com o olhar mais voltado para um HD ativo e também especializado, na literatura específica, estudos de casos e de grupos de indivíduos LHD começaram a ser descritos. O conjunto de sintomas cognitivos, comunicativos e emocionais observado após um acometimento neurológico no HD é denominado por alguns autores de Síndrome do Hemisfério Direito - SHD (Blake, Duffy, Tompkins, \& Myers, 2003; Brookshire, 2003; Bryan, 1995; Marchetti et al., 2005; Morin et al., 2001; Pimental \& Kingsbury, 1989).

Embora maior atenção venha sendo dada aos sinais e sintomas ocasionados por uma lesão de HD há aproximadamente duas décadas, as alterações no processamento cognitivo, comunicativo e emocional associadas a esse acometimento neurológico carecem ainda de mais estudos de revisão e reflexão teórica, assim como de investigações empíricas, que aprimorem sua descrição. A diferença de um século de estudos explica a grande disparidade entre o amplo corpo de conhecimentos sobre o papel do HE na cognição e o restrito corpo de conhecimentos sobre as relações entre o HD e as habilidades cognitivas.

Nesse contexto, o presente ensaio teórico tem como objetivo apresentar uma caracterização da SHD, a partir da busca, na literatura nacional e internacional, de dados acerca das alterações nos processamentos cognitivo, comunicativo e emocional decorrentes de uma lesão de HD. Para tanto, procurarse-á responder às seguintes questões de pesquisa: 1) As alterações cognitivas, comunicativas e/ou emocionais associadas aos quadros de lesão de HD são categorizadas sob quais denominações, além de SHD? 2) Quais as principais alterações cognitivas, comunicativas e emocionais ou comportamentais que ocorrem após uma lesão de HD?

Para responder às questões de pesquisa acima apresentadas, foram investigadas as alterações cognitivas, comunicativas e emocionais ou comportamentais após lesão de HD em algumas obras atuais de Neuropsicologia que dissertam sobre resultados de avaliações neuropsicológicas com indivíduos 
LHD. Além dessa fonte, buscou-se a descrição dessas alterações em manuais de instrumentos de avaliação elaborados especificamente para a população neurológica de LHD e em algumas publicações de periódicos internacionais cujo principal objeto de estudo faz referência direta ou indiretamente à SHD. Não foram encontradas obras nacionais que abordassem o tema em estudo.

No presente ensaio teórico, buscou-se responder às questões de pesquisa através da sistematização de algumas informações obtidas nas obras consultadas (Tabela 1). Cada obra foi caracterizada quanto à categoria da fonte - obra internacional traduzida, obra internacional, instrumento de avaliação elaborado para indivíduos LHD ou artigo científico que abordasse direta ou indiretamente a SHD. Além disso, nessa tabela estão os nomes atribuídos ao conjunto de sinais e sintomas oriundos de acometimento neurológico no $\mathrm{HD}$, assim como quais alterações - cognitivas, comunicativas e/ou emocionais - estão sendo abordadas no quadro pós-lesão de HD em cada obra.

Pode-se observar, com base nos dados apresentados na Tabela 1, que do total de 17 fontes consultadas, o conjunto de sinais e sintomas decorrentes de uma lesão de HD foi denominado de SHD por cinco delas $(29,41 \%)$. Nomes semelhantes, incluindo o termo síndrome, foram encontrados em três fontes estudadas $(17,64 \%)$. O uso do termo déficits foi encontrado em três obras $(17,64 \%)$. As alterações pós-lesão de HD foram denominadas de disfunção em uma obra $(5,89 \%)$. Por fim, apenas uma fonte abordou o termo doença, relacionando os sinais clínicos ao acometimento neurológico propriamente dito $(5,89 \%)$. Quatro fontes $(23,53 \%)$ consultadas não fizeram referência a um nome específico para as sequielas da lesão de $\mathrm{HD}$, embora tenham abordado as alterações funcionais presentes na SHD. Quanto aos déficits mencionados, a maioria apresentou descrição de sinais e sintomas cognitivos, comunicativos e emocionais $(82,35 \%)$, sendo que duas obras abordaram apenas alterações cognitivas $(11,77 \%)$ e uma, somente alterações comunicativas $(5,88 \%)$.

A descrição dos sinais e sintomas presentes em quadros de lesão de HD encontrada nas diferentes obras consultadas será feita em três partes: 1) alterações cognitivas, 2) alterações comunicativas e 3) alterações emocionais. 


\section{Alterações Cognitivas, Comunicativas e Emocionais após Lesão Hemisférica...}

Tabela 1. Dados sobre categoria das fontes, nome atribuído ao quadro pós-lesão de HD e alterações abordadas

\begin{tabular}{|c|c|c|c|}
\hline $\begin{array}{l}\text { Referências das } \\
\text { obras consultadas }\end{array}$ & Categoria da fonte & Nome atribuído & Alterações abordadas \\
\hline $\begin{array}{l}\text { Arroyo, Scrigni, } \\
\text { Bin, Roccatagliata } \\
\text { e Fejerman (1996) }\end{array}$ & Artigo científico & $\begin{array}{c}\text { Disfunção do } \\
\text { hemisfério cerebral } \\
\text { direito }\end{array}$ & $\begin{array}{c}\text { Cognitivas, comunicativas } \\
\text { e emocionais }\end{array}$ \\
\hline Blake et al. (2003) & Artigo científico & SHD & $\begin{array}{c}\text { Cognitivas, comunicativas } \\
\text { e emocionais }\end{array}$ \\
\hline $\begin{array}{c}\text { Botez-Marquard e } \\
\text { Boller (2005) }\end{array}$ & Obra internacional & Ausência de nome & $\begin{array}{c}\text { Cognitivas, comunicativas } \\
\text { e emocionais }\end{array}$ \\
\hline Brookshire (2003) & Obra internacional & SHD & $\begin{array}{c}\text { Cognitivas, comunicativas } \\
\text { e emocionais }\end{array}$ \\
\hline Bryan (1995) & $\begin{array}{l}\text { Instrumento de } \\
\text { avaliação }\end{array}$ & SHD & $\begin{array}{c}\text { Cognitivas, comunicativas } \\
\text { e emocionais }\end{array}$ \\
\hline $\begin{array}{l}\text { Burns, Halper e } \\
\text { Mogil (1985) }\end{array}$ & $\begin{array}{l}\text { Instrumento de } \\
\text { avaliação }\end{array}$ & $\begin{array}{l}\text { Síndromes clínicas } \\
\text { associadas à lesão de } \\
\text { HD }\end{array}$ & $\begin{array}{c}\text { Cognitivas, comunicativas } \\
\text { e emocionais }\end{array}$ \\
\hline Gil (2002) & $\begin{array}{l}\text { Obra internacional } \\
\text { traduzida }\end{array}$ & Ausência de nome & $\begin{array}{c}\text { Cognitivas, comunicativas } \\
\text { e emocionais }\end{array}$ \\
\hline $\begin{array}{l}\text { Joanette, Ska e } \\
\text { Côté (2004) }\end{array}$ & $\begin{array}{l}\text { Instrumento de } \\
\text { avaliação }\end{array}$ & $\begin{array}{c}\text { Déficits cognitivos, } \\
\text { comunicativos e } \\
\text { emocionais dos LHD }\end{array}$ & $\begin{array}{c}\text { Cognitivas, comunicativas } \\
\text { e emocionais }\end{array}$ \\
\hline $\begin{array}{l}\text { Lezak, Howieson e } \\
\text { Loring (2004) }\end{array}$ & Obra internacional & $\begin{array}{c}\text { Déficits característicos } \\
\text { nas lesões de HD }\end{array}$ & $\begin{array}{c}\text { Cognitivas, comunicativas } \\
\text { e emocionais }\end{array}$ \\
\hline Manning (2005) & Obra internacional & Ausência de nome & Cognitivas \\
\hline Morin et al. (2001) & Artigo científico & SHD & $\begin{array}{c}\text { Cognitivas, comunicativas } \\
\text { e emocionais }\end{array}$ \\
\hline Myers (1999) & Obra internacional & $\begin{array}{c}\text { Déficits associados à } \\
\text { lesão de HD }\end{array}$ & $\begin{array}{c}\text { Cognitivas, comunicativas } \\
\text { e emocionais }\end{array}$ \\
\hline Myers (2001) & Artigo científico & $\begin{array}{l}\text { Sindrome da lesão do } \\
\text { HD }\end{array}$ & Comunicativas \\
\hline $\begin{array}{c}\text { Pimental e } \\
\text { Kingsbury (1989) }\end{array}$ & $\begin{array}{l}\text { Instrumento de } \\
\text { avaliação }\end{array}$ & Síndromes do HD & $\begin{array}{c}\text { Cognitivas, comunicativas } \\
\text { e emocionais }\end{array}$ \\
\hline $\begin{array}{l}\text { Snyder, Nussbaum } \\
\text { e Robins (2006) }\end{array}$ & Obra internacional & $\begin{array}{c}\text { Doença } \\
\text { cerebrovascular do HD }\end{array}$ & Cognitivas \\
\hline $\begin{array}{l}\text { Springer e Deutsch } \\
\qquad(1998)\end{array}$ & $\begin{array}{l}\text { Obra internacional } \\
\text { traduzida }\end{array}$ & Ausência de nome & $\begin{array}{c}\text { Cognitivas, comunicativas } \\
\text { e emocionais }\end{array}$ \\
\hline $\begin{array}{l}\text { Venegas et al. } \\
\text { (1998) }\end{array}$ & Artigo científico & SHD & $\begin{array}{c}\text { Cognitivas, comunicativas } \\
\text { e emocionais }\end{array}$ \\
\hline
\end{tabular}




\section{Alterações cognitivas}

A SHD é, em geral, caracterizada por englobar distúrbios nas funções de orientação temporo-espacial, atenção, percepção, memória visual, habilidades aritméticas e praxia construtiva (Brookshire, 2003; Blake et al., 2003). Disfunções executivas também são referidas (Annoni et al., 2003).

A orientação quanto ao tempo e ao espaço pode estar prejudicada na síndrome em estudo, principalmente o componente espacial (Bryan, 1995; Pimental \& Kingsbury, 1989). Na desorientação visuo-espacial, salienta-se que o distúrbio de desorientação geográfica é menos comum do que o de desorientação topográfica. O primeiro diz respeito ao reconhecimento mínimo da natureza do ambiente ao redor do indivíduo LHD, não acompanhado pelo reconhecimento da sua localização específica. A pessoa com desorientação geográfica sabe orientar-se no tempo e na diferenciação entre si e as outras pessoas; não sabe, porém, onde está. A desorientação topográfica caracteriza-se pela dificuldade que os LHD apresentam em se relacionar com o espaço externo, pois este não está representado internamente. Fracassam, muitas vezes, em seguir rotinas familiares e ler mapas, provavelmente por não reconhecerem pistas familiares (Brookshire, 2003). As lesões vasculares são geralmente na região medial do lobo occipital e/ou no giro para-hipocampal direitos, assim como na substância branca adjacente ao território da artéria cerebral posterior. Apesar de a maioria dos autores fazerem referência a distúrbios dessa função, há alguns estudos que não evidenciaram diferenças entre indivíduos LHD e controles em tarefas de orientação espacial (por exemplo, Harvey et al., 2002).

Além do déficit de orientação, a SHD também abrange distúrbios atencionais. Os processos de atenção prejudicados podem embasar, pelo menos parcialmente, a severidade das demais seqüelas cognitivas e comportamentais, já que essa é uma função cognitiva básica para os outros processamentos (Ishiai, 1997). Muitos indivíduos LHD apresentam dificuldade em tarefas diárias que demandam atenção mantida - focalização da atenção em um estímulo por um determinado período de tempo - e dividida - atenção distribuída entre dois ou mais estímulos concomitantes (Myers, 1999). Assim, por exemplo, não têm êxito completo em apreender o sentido geral dos eventos e em manter padrões apropriados de interação com interlocutores. 
Alterações Cognitivas, Comunicativas e Emocionais após Lesão Hemisférica...

Outra função que geralmente está afetada na SHD é a percepção. Pimental e Kingsbury (1989) referem que as alterações perceptivas presentes nessa síndrome envolvem, com freqüência, componentes visuo-espaciais, englobando, assim, distúrbios do esquema corporal, da percepção de cores provenientes do hemicampo contralateral à lesão (acromatopsia) e da discriminação entre direita e esquerda. Em complementaridade, Brookshire (2003) e Burns et al. (1985) enfatizam que os distúrbios perceptivos da SHD abrangem a ocorrência de anosognosia, heminegligência sensorial e prosopagnosia.

A anosognosia corresponde à ausência de uma consciência plena dos déficits ou, ainda, da amplitude do impacto funcional desses na vida do indivíduo LHD (Joanette, Ska, \& Côté, 2004; Rode et al., 2005). O não reconhecimento das sequielas da lesão cerebral é muito comum após lesão de HD, principalmente do lobo parietal (Léger, 2005). O indivíduo LHD tende a não reconhecer sua hemiparesia contralateral e sua alteração de percepção visual, por exemplo. Brookshire (2003) e Joanette et al (2004) salientam que há diferentes formas de anosognosia, de acordo com o nível de intensidade desse distúrbio.

Geralmente associada à anosognosia, a heminegligência sensorial ou espacial esquerda é um distúrbio em que o indivíduo lesado pode não perceber estímulos sensoriais, tais como táteis, auditivos e/ou visuais, provenientes do campo espacial esquerdo (Dauriac-Le et al., 2002; Lezak, Howieson, \& Loring, 2004; Manning, 2005). Alguns sintomas freqüentes são ausência de reação a pessoas, sons e objetos localizados à esquerda da linha média corporal; realização de cuidados pessoais apenas no lado direito do corpo; falha no movimento dos membros esquerdos superior e inferior; colisão em paredes e portas à esquerda; leitura e escrita restritas à metade direita da página; e, desinteresse pela participação em programas de reabilitação. Esse distúrbio pode ser acarretado por um prejuízo na representação mental das relações visuo-espaciais e/ou por um prejuízo nos processamentos atencional e perceptivo. Há, no entanto, controvérsias na literatura quanto à qual função cognitiva está alterada nesses quadros. Alguns autores defendem a hipótese de que a anosognosia e a heminegligência tratam-se de distúrbios atencionais (Deouell, Sacher, \& Soroker, 2005; Verfaellie \& Heilman, 2006), enquanto outros acreditam que tais quadros envolvem déficits do processamento perceptivo (por exemplo, $\mathrm{Lu}$, Ye, Zhou, Lu, \& Chen, 2005). 
Quanto aos correlatos neurais da anosognosia e da heminegligência sensorial, o lobo parietal direito é a associação mais mencionada na literatura (Léger, 2005). Além disso, lesões de estruturas subcorticais, tal como o tálamo, também são referidas (Rainville, Giroire, Periot, Cuny, \& Mazaux, 2003). No entanto, tais distúrbios também são associados a lesões esquerdas. Léger (2005) salienta que a heminegligência pode estar presente em indivíduos com lesão de HE ou HD, sendo, porém, muito mais freqüente na segunda população neurológica. Em estudos comparativos de grupos LHD e lesados de HE (LHE), geralmente ao redor do dobro de LHD com relação aos indivíduos LHE apresenta heminegligência em tarefas de percepção visual. Por exemplo, Deouell et al., (2005) constataram que 66\% dos pacientes LHD avaliados apresentou tempo de reação e acurácia significativamente inferiores em provas de detecção de estímulos visuais, enquanto $31 \%$ dos LHE demonstrou déficits contralaterais significativos.

A prosopagnosia também é uma alteração perceptiva da SHD. Esse distúrbio consiste na inabilidade de reconhecer faces de pessoas familiares, tais como de amigos e parentes (Burns et al., 1985; Wilkinson, Ko, Kilduff, McGlinchey, \& Milberg, 2005). Assim, os LHD demonstram pouca habilidade em reconhecer faces previamente apresentadas, assim como em reconhecer pessoas famosas através de fotografias. Os déficits de percepção de faces, segundo Bryan (1995), não são restritos aos estímulos faciais familiares, mas abrangem, também, o reconhecimento problemático de faces não familiares. De tal modo, os indivíduos LHD podem não ser eficazes em tarefas de combinar ou associar faces semelhantes.

Além de distúrbios atencionais e perceptivos, os pacientes LHD também podem apresentar alterações de memória não-verbal. A maior dificuldade dos LHD está em armazenar e evocar estímulos não-verbais, apresentados visual ou auditivamente, sendo mais problemáticos os estímulos visuais (Burns, et al., 1985; Manning, 2005). Ferber e Danckert (2006) evidenciaram que indivíduos LHD também apresentam déficit de armazenamento e recuperação de informações espaciais, agravado pela heminegligência. Adicionalmente, também é referido na literatura um distúrbio da memória de trabalho espacial em LHD. As lesões de HD em pacientes com déficits mnemônicos geralmente localizamse no lobo parietal e/ou na insula (Malhotra et al., 2005; Pisella, Berberovic, \& Mattingley, 2004). Há casos de lesões parieto-temporais (Léger, 2005). 
Alterações Cognitivas, Comunicativas e Emocionais após Lesão Hemisférica...

Outro quadro de alteração mnemônica associado à SHD é a paramnésia reduplicativa ou reduplicação ambiental (Burns, et al., 1985; Pimental \& Kingsbury, 1989). Esse déficit caracteriza-se pela insistência do indivíduo em afirmar que um local novo, como o hospital, está localizado próximo a outro local que lhe é muito familiar, como sua residência. A lesão parietal direita está muito correlacionada a esse sintoma.

O processamento aritmético pode, também, estar prejudicado na SHD. A acalculia ou discalculia - prejuízo na habilidade de realizar operações aritméticas - está associada, muitas vezes, a lesões nas regiões pós-rolândicas, principalmente parieto-occiptal, do HD, apesar de que lesões bilaterais também são observadas (Gil, 2002). A discalculia em indivíduos LHD pode ser secundária às alterações visuo-espaciais, podendo ser denominada discalculia espacial, na qual o indivíduo apresenta dificuldades em calcular operações no papel e/ou mentalmente (Léger, 2005; Levin et al., 1996).

Com maior freqüência do que a discalculia, os distúrbios práxicos fazem parte das descrições da SHD. Uma dispraxia consiste na falta de habilidade para realizar movimentos aprendidos ou intencionais, apesar de não haver paralisia ou perda sensorial (Manning, 2005). A apraxia ideomotora - dificuldade no planejamento do movimento intencional - está mais relacionada a lesões de HE, enquanto a apraxia construtiva - prejuízo da habilidade de reproduzir ou construir figuras, desenhando ou montando - a lesões de HD (Heilman, Watson, \& Gonzales-Rothi, 2006). Outra dispraxia que pode fazer parte da SHD é a do vestir-se (Burns et al, 1995; Pimental \& Kingsbury, 1989). Gil (2002) justifica essa dispraxia pelo fato de a região posterior do HD ser o local de convergência das informações visuais, espaciais e motivacionais necessárias para a atividade do vestir-se. Uma pessoa que tem lesão nessa região observa sua roupa, vira-a e revira-a, e, por fim, desiste de vesti-la ou a coloca de modo mal-sucedido. Além dessas alterações práxicas, Spinazzola, Cubelli e Della Sala (2003) referem maior frequiência de reações de instabilidade postural em participantes LHD do que nos LHE.

Por fim, no que concerne às funções executivas, alguns dos seus componentes podem estar alterados na população neurológica de LHD, levando a uma síndrome disexecutiva severa (Rainville et al., 2003). A impulsividade, que denota déficits de inibição, é referida pelos autores (por exemplo, 
Brookshire, 2003). Os indivíduos LHD tendem a responder aos estímulos rápida e impulsivamente, aumentando seu percentual de erros em tarefas neuropsicológicas. Dentre as tarefas que avaliam componentes das funções executivas, a fluência verbal apresenta-se geralmente alterada em indivíduos LHD, após lesões corticais ou talâmicas (Annoni et al., 2003). Quanto às habilidades de planejamento e resolução de problemas, Colvin, Dunbar e Grafman (2001) constataram que pacientes LHD foram significativamente piores em tarefas que avaliam essas funções quando comparados aos controles, mas melhores quando a base de comparação era pacientes LHE e lesados bilaterais.

Em suma, o indivíduo LHD caracteriza-se por apresentar um conjunto de sinais e sintomas cognitivos, dentre os quais podem ser mencionados, principalmente, desorientação topográfica, anosognosia e heminegligência, dismnésia não-verbal e visual, discalculia, dispraxia construtiva e disfunção executiva. No entanto, Brookshire (2003) salienta que nem todos LHD apresentam os sintomas cognitivos acima referidos, havendo uma grande variabilidade na manifestação da SHD.

\section{Alterações comunicativas}

Além das alterações cognitivas, distúrbios comunicativos também são descritos como sintomas da SHD (Blake et al., 2003; Morin et al., 2001; Myers, 2001). As alterações na função comunicativa mais associadas às lesões de HD são déficits na compreensão e na produção de discurso, na habilidade de processar inferências (a partir de informações explícitas, apreender as implícitas), na capacidade de interpretar significados ambíguos, no processamento de informações contextuais e na compreensão e produção prosódicas (Myers, 1999). Enfim, os LHD podem apresentar déficits em quatro processamentos comunicativos: discursivo, pragmático-inferencial, léxico-semântico e prosódico.

Nesse contexto, pode-se evidenciar nos LHD dificuldades de processamento do discurso conversacional, com prejuízo da troca de turnos, da manutenção do assunto, de habilidades pragmático-discursivas, tais como apreender a intenção do interlocutor e conduzir o discurso com base nela (Kasher, Batori, Soroker, Gravers, \& Zaidel, 1999) e de uso adequado de referências (Chantraine, Joanette, \& Ska, 1998). No discurso narrativo, também são mencionados 
Alterações Cognitivas, Comunicativas e Emocionais após Lesão Hemisférica...

déficits: os LHD apresentam maior dificuldade em construir histórias a partir de estímulos visuais desordenados (Marini, Carlomagno, Caltagirone, \& Nocentini, 2005), além de déficit no reconto de histórias, com omissão de informações importantes para a compreensão do texto (Bartels-Tobin \& Hinckley, 2005).

No que concerne ao processamento pragmático-inferencial, os pacientes com lesão de HD podem ter dificuldades em compreender sentenças metafóricas (Champagne, Desauels, \& Joanette, 2003). Além disso, tendem a apresentar maior dificuldade do que indivíduos controles em interpretar atos de fala indiretos e em produzir pedidos baseados nos objetivos do interlocutor (McDonald, 2000).

Quanto ao processamento léxico-semântico, as habilidades de evocação lexical (Beausoleil, Fortin, Le Blanc, \& Joanette, 2003) e de julgamento semântico podem estar alteradas (Nocentini, Goulet, Roberts, \& Joanette, 2001). Dessa forma, os indivíduos LHD tendem a produzir menor quantidade de vocábulos que sujeitos controles, assim como se desempenham pior na identificação de relação semântica entre palavras, respectivamente.

Por fim, os processamentos prosódicos lingüístico (entonação dada para diferenciar interrogações de afirmações, por exemplo) e emocional (entonação que distingue diferentes sentimentos) estão, em geral, prejudicados na SHD. Assim sendo, os LHD desempenham-se pior do que os LHE e os controles em tarefas de discriminação, imitação e produção de sentimentos de medo, tristeza e raiva (Charbonneau, Scherzer, Aspirot, \& Cohen, 2003).

Em suma, as alterações comunicativas decorrentes de um acometimento neurológico no HD não englobam alterações dos aspectos formais da linguagem (processamento de sons, palavras e sentenças), mas sim dos seus aspectos funcionais comunicativos (processamento semântico de informações não literais, processamentos pragmático e prosódico). Por tal motivo, autores como Joanette et al (2004) defendem o uso do termo déficit comunicativo na SHD em oposição ao déficit lingüístico, tradicionalmente presente nos quadros de afasia, caracterizados por uma alteração do processamento da linguagem após uma lesão de HE. Com referência aos correlatos neurais das alterações comunicativas da SHD, há descrição de diferentes regiões corticais e subcorticais direitas. A área mais mencionada corresponde à região análoga às áreas de linguagem do HE (Wildgruber, 2005). 
Na literatura, nota-se que apenas $50 \%$ dos participantes dos grupos de lesados apresenta alterações no processamento comunicativo, com desempenho prejudicado em habilidades distintas quando comparados entre si (Brookshire, 2003; Joanette et al., 2004). Essa heterogeneidade também é estendida para as alterações emocionais.

\section{Alterações emocionais}

As alterações emocionais são abordadas sob diferentes denominações nas obras consultadas. Pimental e Kingsbury (1989), por exemplo, descrevem os distúrbios da emoção e do afeto, dentre os quais incluem os déficits de percepção e expressão das emoções transmitidas pela mímica facial. As alterações emocionais e afetivas referidas por Myers (1999), além do processamento emocional de faces alterado, abrangem o prejuízo na compreensão e na expressão de estímulos prosódicos com entonação emocional. Pode-se observar, assim, que o processamento prosódico emocional ineficaz pode ser considerado tanto como uma alteração comunicativa como uma alteração afetiva.

Além disso, as alterações emocionais da SHD também incluem mudanças comportamentais, que podem ser consideradas distúrbios neuropsiquiátricos. Podem ser observados, então, em quadros de SHD, sintomas tais como confusão, agitação, delírio, estados de paranóia, alucinações visuais, mania secundária, síndromes de identificação inadequada, entre outros (Allegri et al., 1996; Burns et al., 1985; Myers, 1999). Quanto aos comportamentos sociais, os LHD tendem a apresentar desempenho prejudicado na produção de respostas de empatia. $\mathrm{O}$ correlato neural desse déficit afetivo é o córtex ventromedial direito (Shamay-Tsoory, Tomer, Berger, \& Aharon-Peretz, 2003).

Assim sendo, além dos componentes cognitivos e comunicativos, fazem parte da SHD, ainda, componentes sócio-afetivos. Esses dizem respeito tanto ao processamento emocional prosódico e facial, como a alterações neuropsiquiátricas. 
Alterações Cognitivas, Comunicativas e Emocionais após Lesão Hemisférica...

\section{Caracterização da SHD: pontos de reflexão}

No processo de busca de uma caracterização da SHD, observou-se falta de consenso na literatura quanto ao termo utilizado para nomear o conjunto de sinais e sintomas presentes após uma lesão de HD. A denominação SHD, apesar de ter sido a mais mencionada nas obras consultadas, não é reconhecida com unanimidade, sendo ainda utilizados vários termos para definir as alterações cognitivas, comunicativas e emocionais presentes no quadro neurológico em pauta. Em contrapartida, os déficits cognitivos e principalmente comunicativos associados a lesões de HE são bem reconhecidos pelos pesquisadores. Por exemplo, a dispraxia ou apraxia ideomotora e a afasia correspondem a termos de consenso na literatura. Como há mais de um século de diferença entre o interesse científico pelo $\mathrm{HE}$ e o interesse pelo $\mathrm{HD}$, provavelmente a quantidade e a variedade dos estudos realizados envolvendo a população de LHD ainda não foram suficientes para que um único termo se difundisse consensualmente entre os diferentes grupos de pesquisa.

Além desse fator histórico, a noção de síndrome na neuropsicologia pode ser outro fator que contribuiu para a falta de consenso evidenciada. Siksou (2005) ressalta que a noção de síndrome para a Neuropsicologia engloba relações de associação entre sinais, mas não necessariamente de causalidade. Além disso, a autora critica a utilização exclusiva da noção de síndrome, que preconiza o enquadramento dos sintomas do paciente em uma determinada patologia, sem considerar as especificidades de cada caso. Dessa maneira, o termo síndrome pode não estar sendo utilizado por alguns neuropsicólogos pesquisadores por representar uma generalização de casos com distintas manifestações dos sintomas associados à SHD, além de haver uma relação de causalidade implícita entre acometimento neurológico do HD e os déficits cognitivos, comunicativos e emocionais abordados.

No entanto, como a identificação de uma síndrome apresenta um valor preditivo importante, a continuidade do processo de difusão do uso do termo SHD e dos sintomas a ela associados é válida. Blake et al. (2003) ressaltam que a ausência de uma terminologia comum dificulta a comunicação científica e clínica. O agrupamento de sinais e sintomas sob o nome de um determinado quadro facilita a identificação e o reconhecimento da sua manifestação pelos diferentes profissionais. Esse processo de identificação e reconhecimento de 
uma entidade clínica, porém, requer critérios diagnósticos explícitos e distintivos (Gagnon, Mottron, \& Joanette, 1997). Como para muitos sinais e sintomas após uma lesão de HD, os estudos ainda são pouco freqüentes, tais como memória, habilidades aritméticas e funções executivas, uma maior quantidade de pesquisas é necessária para que critérios diagnósticos claros e precisos sejam estabelecidos. Não se pode, entretanto, desconsiderar a importância crescente do termo SHD na literatura recente, ilustrada em uma investigação sobre SHD cruzada - sintomas típicos de lesão de HD presentes pós-lesão de HE (Marchetti et al., 2005). O uso desse termo demonstra uma aproximação cada vez maior com o nível de importância do termo afasia para os quadros de lesão de HE, utilizado na dissociação análoga "afasia cruzada".

Em relação à busca das principais alterações cognitivas, comunicativas e emocionais que ocorrem após uma lesão de HD, dois aspectos serão discutidos: 1) predominância da descrição de sintomas atencionais e perceptivos e 2) heterogeneidade da manifestação da SHD. Quanto ao primeiro aspecto, observou-se que os quadros de heminegligência e de anosognosia são abordados com maior frequiência e profundidade do que os demais aspectos cognitivos e do que os déficits comunicativos e emocionais. A heminegligência é, até mesmo, considerada uma síndrome independente por alguns autores: síndrome da heminegligência (Rode et al., 2005; Verfaellie \& Heilman, 2006, por exemplo).

Além da alta prevalência, esses dois quadros são muito associados a lesões de HD porque prejudicam significativamente a rotina diária do indivíduo LHD, ocasionando um importante handicap e um prejuízo na qualidade de vida. Adicionalmente, nos casos em que a heminegligência e/ou a anosognosia estão presentes, provavelmente esses quadros de alteração atencional-perceptiva podem agravar os demais déficits cognitivos, comunicativos e comportamentais. Desse modo, tendem a aumentar a severidade da SHD.

Embora sejam muito prevalentes e importantes pelos déficits funcionais que acarretam no indivíduo LHD, o foco predominante nesses sintomas não é suficiente para a compreensão da SHD como um todo. Há, inclusive, muitos casos de pacientes com déficits de memória, praxias, comunicação, comportamento sócio-afetivo sem a comorbidade anosognosia ou heminegligência, evidenciando uma dissociação entre esses déficits (Deouell et al., 
Alterações Cognitivas, Comunicativas e Emocionais após Lesão Hemisférica...

2005). Tal dissociação dá força à noção de que as demais alterações não são meras conseqüências da falta de consciência do distúrbio ou da negligência dos estímulos contralaterais.

No que concerne ao segundo aspecto de reflexão evidenciado no processo de busca das principais alterações cognitivas, comunicativas e emocionais que ocorrem após uma lesão de HD - heterogeneidade da manifestação da SHD - algumas hipóteses são levantadas. Primeiramente, cada alteração cognitiva pode apresentar subtipos de manifestação. Subtipos de desorientação topográfica, anosognosia e de heminegligência já vêm sendo abordados na literatura (Buxbaum et al., 2004; Léger, 2005). O desconhecimento de alguns subtipos pode obscurecer o processo de avaliação neuropsicológica do processamento de cada função cognitiva na população neurológica específica de LHD. Em segundo lugar, levanta-se a hipótese de que os métodos de avaliação neuropsicológica usados em alguns estudos podem não ser os mais sensíveis à detecção de um déficit na função avaliada. As diferenças metodológicas nos estudos com LHD podem, então, explicar, pelo menos em parte, falsos negativos. Isso porque algumas tarefas são mais sensíveis do que outras para identificar um determinado sintoma. No caso da verificação da heminegligência, por exemplo, Milner e McIntosh (2005) constataram que as tarefas de cancelamento avaliam mais os aspectos centrais da heminegligência do que as provas de bissecção de linhas, mais utilizadas que as primeiras.

A terceira hipótese para explicar essa heterogeneidade diz respeito, ainda, aos aspectos metodológicos dos estudos, mas não especificamente aos testes neuropsicológicos utilizados, que são muito distintos entre as diferentes investigações. Sucintamente, a variabilidade de resultados pode se dever à inclusão de indivíduos LHD com diferentes locais e extensões de lesão e/ou com diferentes graus de severidade do distúrbio neurológico e tempo pós-lesão, com recuperação espontânea completada ou não, e diferentes níveis de escolaridade na mesma amostra (Tompkins, Fassbinder, Lehman-Blake, \& Baumgaertner, 2002). O controle de todas as variáveis entre-sujeito é praticamente impossível quando a amostra é composta por um grupo clínico de LHD.

Com base nos aspectos acima discutidos, devem-se evitar generalizações e uma visão estereotipada do indivíduo LHD, uma vez que provavelmente há subclassificações da SHD, ainda a serem descobertas e melhor investi- 
gadas, com maior casuística. Talvez essa tendência de generalizar aqueles distúrbios apresentados apenas por alguns indivíduos para todos os acometidos por lesão de HD seja uma maneira de se tentar agrupar o conjunto de sintomas encontrados após a lesão nesse hemisfério de forma semelhante à taxonomia das afasias, distúrbio associado ao HE. Na classificação das afasias, a heterogeneidade foi amenizada pelas subclassificações.

\section{Considerações finais}

A SHD é uma neuropsicopatologia que necessita, ainda, de muitos estudos teóricos e empíricos, envolvendo estudos de casos e de grupos, para ser melhor compreendida. Sua caracterização é muito importante para uma comunicação efetiva entre neuropsicolólogos pesquisadores e clínicos. Nesse processo de busca por uma caracterização, a generalização não se mostra como a melhor solução, já que nem todos os indivíduos LHD apresentam déficits e aqueles que apresentam nem sempre possuem todas as alterações cognitivas, comunicativas e emocionais abordadas nesse ensaio teórico. Uma maior quantidade de investigações com amostras de LHD examinando com profundidade o processamento de cada função cognitiva e comunicativa e dos diferentes comportamentos sócio-afetivos é necessária para uma adequada delimitação dos prováveis subtipos e perfis da síndrome.

Para que a descrição inconsistente de seqüelas cognitivas, comunicativas e comportamentais após lesão de HD possa ser melhor entendida, o estudo sobre tais seqüelas deve ser conduzido com alto rigor metodológico por clínicos e pesquisadores em Neuropsicologia. O controle cuidadoso de variáveis entre e intra-sujeito é essencial para que análises comparativas possam evidenciar predominantemente o efeito da variável lesão de HD, embora um controle ideal seja utópico. O papel do HD no comportamento humano de um modo geral precisa ser melhor investigado, tanto em indivíduos neurologicamente preservados quanto em LHD, possibilitando que um aprofundamento teóricometodológico das pesquisas sobre a SHD tornem-na mais conhecida nacional e internacionalmente.

O aumento do interesse em se estudar mais detalhadamente a SHD refletirá em implicações para o diagnóstico, o prognóstico e a reabilitação de 
indivíduos LHD. Conseqüentemente, ocorrerão melhorias para a rotina diária, a interação social e a qualidade de vida dessa população.

Fonseca, R. P., Ferreira, G. D., Liedtke, F. V., Muller, J. L., Sarmento, T. F., \& Parente, M. A. M. P. (2006). Cognitive, communicative and emotional deficits after a right hemispheric lesion: Looking for the features of the Right Hemisphere Syndrome. Psicologia USP, 17(4), 241-262.

\begin{abstract}
The group of signs and symptoms observed after a right hemisphere damage can be called Right Hemisphere Syndrome - RHS. This paper aims to present a description of the mentioned neuropsychological pathology. Cognitive deficits, such as disorders of attention and perception (anosognosia, hemineglect and prosopagnosia), memory (visual and spatial amnesia and working memory disorder), praxis (constructional disability) and executive functions (executive dysfunctions) characterize the RHS. When it comes to communicative abilities, the RHS is characterized by discoursive, pragmatic, lexicalsemantic and prosodic deficits. Emotional disorders include inaccurate emotions comprehension and production from facial expressions and vocal intonation; besides these deficits, there are neuropsychiatric symptoms. However, a great heterogeneity in RHS manifestation is observed. For a deeper knowledge of RHS, it is necessary to promote more single case and group studies with the right brain damaged population.
\end{abstract}

Index Terms: Neuropsychology. Cognition. Communication. Emotion. Right hemisphere.

Fonseca, R. P., Ferreira, G. D., Liedtke, F. V., Muller, J. L., Sarmento, T. F., \& Parente, M. A. M. P. (2006). Déficits cognitifs, communicatifs et émotionnels après une lésion de l'hémisphère droit: en quête des caractéristiques de la Syndrome de l'Hémisphère Droit. Psicologia USP, 17(4), 241-262.

Résumé: L'ensemble des signes et symptomes observés après une atteinte cérébrale droite peut être nommé de Syndrome de l'Hémisphère Droit - SHD. Cet article vise présenter une caractérisation de cette 
syndrome. Les altérations cognitives, comme des déficits de l'attention, perception, mémoire, praxies et fonctions executives, avec la présence de l'anosognosie, héminégligence spatiale, prosopagnosie, altérations de la mémoire spaciale et de travail, apraxie constructive et disfonctions executives, principalement les altéracions perceptives, sont celles les plus décrites. Séquelles dans toutes les fonctions cognitives et dans les processus communicatifs - atteintes discursives, pragmatiques, lexico-sémantiques et prosodiques - et émotionnels - difficultés de la compréhension et production des expressions émotionnelles faciales et vocales - sont considérées comme partie de la SHD même s'il y a une hétérogéneité quand elle se manifeste. Il devrait avoir plus d'études de cas et de groupe avec les cérébrolésés droits pour qu'on puisse meilleur comprendre la SHD.

Mots-clés: Neuropsychologie. Cognition. Communication. Émotion. Hémisphère droit.

\section{Referências}

Allegri, R. F., Elli, J., Valicenti, M. R., Mangone, C. A., Taragano, F. E., \& Ranalli, C. G. (1996). Síndromes neuropsiquiátricos por lesión en el hemisferio cerebral derecho. Acta Psiquiátrica y Psicológica de América Latina, 42(3), 222-229.

Annoni, J. M., Khateb, A., Gramigna, S., Staub, F., Carota, A., Maeder, P. et al. (2003). Chronic cognitive impairment following laterothalamic infarcts: A study of 9 cases. Archives of Neurology, 60(10), 1439-1443.

Arroyo, H. A., Scrigni, A., Bin, L., Roccatagliata, G., \& Fejerman, N. (1996). Disfunción aguda del hemisferio cerebral derecho e hipocomplementemía. Archivos Argentinos de Pediatría, 94(1), 49-55.

Bartels-Tobin, L. R., \& Hinckley, J. L. (2005). Cognition and discourse production in right hemisphere disorder. Journal of Neurolinguistics, 18(6), 461-477.

Beausoleil, N., Fortin, R., Le Blanc, B., \& Joanette, Y. (2003). Unconstrained oral naming performance in right-and left-hemisphere-damaged individuals: When education overrides the lesion. Aphasiology, 17, 143-158.

Blake, M. L., Duffy, J. R., Tompkins, C. A., \& Myers, P. S. (2003). Right hemisphere syndrome is in the eye of the beholder. Aphasiology, 17(5), 423-432.

Botez-Marquard, T., \& Boller, F. (2005). Neuropsychologie clinique et neurologie du comportement. Montréal: Les Presses de 1’Université de Montréal. 
Alterações Cognitivas, Comunicativas e Emocionais após Lesão Hemisférica...

Brookshire, R. H. (2003). Introduction to neurogenic communication disorders. Missouri: Mosby.

Bryan, K. L. (1995). The right hemisphere language battery (2nd ed.). England: Whurr.

Burns, M. S., Halper, A. S., \& Mogil, S. I. (1985). Clinical management of right hemisphere dysfunction. Chicago: Aspen.

Buxbaum, L. J., Ferraro, M. K., Veramonti, T., Farne, A., Whyte, J., Ladavas, E. et al. (2004). Hemispatial neglect: Subtypes, neuroanatomy, and disability. Neurology, 62(5), 749-756.

Champagne, M., Desautels, M., \& Joanette, Y. (2003). Accounting for the pragmatic déficit in RHD individuals: A multiple case study. Brain and Language, 87, 210-211.

Chantraine, Y., Joanette, Y., \& Ska, B. (1998). Conversational abilities in patients with right hemisphere damage. Journal of Neurolinguistics, 11(1-2), 21-32.

Charbonneau, S., Scherzer, B. P., Aspirot, D., \& Cohen, H. (2003). Perception and production of facial and prosodic emotions by chronic CVA patients. Neuropsychologia, 41, 605-613.

Colvin, M. K., Dunbar, K., \& Grafman, J. (2001). The effects of frontal lobe lesions on goal achievement in the water jug task. Journal of Cognitive Neuroscience, 13(8),1129-1147.

Dauriac-Le, M. V., Mailhan, L., Louis-Dreyfus, A., De, M. G., Denys, P., Bussel, B. et al. (2002). Double dissociation entre négligence unilatérale gauche et anosognosie. Revue neurologique, 158(4), 427-430.

Deouell, L. Y., Sacher, Y., \& Soroker, N. (2005). Assessment of spatial attention after brain damage with a dynamic reaction time test. Journal of the InternationalNeuropsychological Society, 11(6), 697-707.

Ferber, S., \& Danckert, J. (2006). Lost in space - the fate of memory representations for nonneglected stimuli. Neuropsychologia, 44(2), 320-325.

Gagnon, L., Mottron, L., \& Joanette, Y. (1997). Questioning the validity of the semanticpragmatic syndrome diagnosis. Autism, 1(1), 37-55.

Gil, R. (2002). Neuropsicologia. São Paulo: Santos.

Harvey, M., Jackson, S. R., Newport, R., Krämer, T., Morris, D. L., \& Dow, L. (2002). Is grasping impaired in hemispatial neglect? Behavioral Neurology Unit, 13(1-2), 17-28.

Heilman, K. M., Watson, R. T., \& Gonzales-Rothi, L. J. (2006). Limb apraxias. In P. J. Snyder, P. D. Nussbaum \& D. L. Robins (Eds.), Clinical neuropsychology (pp. 534546). Washington: APA.

Ishiai S. (1997). Neuropsychological disorders in minor hemisphere damage. Clinical Neurology, 37(12), 1122-1124. 
Joanette, Y., Ska, B., \& Côté, H. (2004). Protocole MEC - Protocole Montreál d'Évaluation de la Communication. Montreal: Ortho Édition.

Karussis, D., Leker, R. R., \& Abramsky, O. L. (2000). Cognitive dysfunction following thalamic stroke: A study of 16 cases and review of the literature. Journal of the Neurological Sciences, 172, 25-29.

Kasher, A., Batori, G., Soroker, N., Graves, D., \& Zaidel, E. (1999). Effects of right- and lefthemisphere damage on understanding conversational implicatures. Brain and Language, 68(3), 566-590.

Léger, G.C.(2005).Le syndrome parietal.InT.Botez-Marquard \& F. Boller, Neuropsychologie clinique et neurologie du comportement (pp. 167-194). Montréal: Les Presses de 1’Université de Montréal.

Levin, H. S., Scheller, J., Rickard, T., Grafman, J., Martinkowski, K., Winslow, M. et al. (1996). Dyscalculia and dyslexia after right hemisphere injury in infancy. Archives of Neurology, 53(1), 88-96.

Lezak, M. D., Howieson, D. B., \& Loring, D. W. (2004). Neuropsychological assessment. New York: Oxford Univertsity Press.

Lu, X. S., Ye, J., Zhou, S., Lu, B. X., \& Chen, X. H. (2005). Unilateral spatial neglect, global processing deficit and prosopagnosia following right hemisphere stroke: A case report. Chinese Medical Journal, 118(21), 1846-1848.

Malhotra, P., Jäger, H. R., Parton, A., Greenwood, R., Playford, E. D., Brown, M. M. et al. (2005). Spatial working memory capacity in unilateral neglect. Brain, 128(2), 424-435.

Manning, L. (2005). La neuropsychologie clinique. Paris: Armand Colin.

Marchetti, C., Carey, D., \& Della Sala, S. (2005). Crossed right hemisphere syndrome following left thalamic stroke. Journal of Neurology, 252(4), 403-411.

Marini, A., Carlomagno, S., Caltagirone, C., \& Nocentini, U. (2005). The role played by the right hemisphere in the organization of complex textual structures. Brain and Language, 93, 46-54.

McDonald, S. (2000). Exploring the cognitive basis of right-hemisphere pragmatic language disorders. Brain and Language, 75, 82-107.

Milner, A. D., \& McIntosh, R. D. (2005). The neurological basis of visual neglect. Current Opinion in Neurology, 18(6), 748-753.

Morin, C., Taillefer, C., Vallat, C., Helsly, N., Thibierge, S., \& Pradat-Diehl, P. (2001). Qu'estce qu'un gauche? Annales de réadaptation et de médecine physique, 44(4), 192-204.

Myers, P. S. (1999). Right hemisphere damage. San Diego, CA: Singular. 
Alterações Cognitivas, Comunicativas e Emocionais após Lesão Hemisférica...

Myers, P. S. (2001). Toward a definition of RHD syndrome. Aphasiology, 15(10/11), 913-918.

Nocentini, U., Goulet, P., Roberts, P. M., \& Joanette, Y. (2001). The effects of left- versus right-hemisphere lesions on the sensitivity to intra- and interconceptual semantic relationships. Neuropsychogia, 39, 443-451.

Pimental, P. A., \& Kingsbury, N. A. (1989). Mini Inventory of Right Injury. Austin: TX: PRO-ED.

Pisella, L., Berberovic, N., \& Mattingley, J. B. (2004). Impaired working memory for location but not for colour or shape in visual neglect: A comparison of parietal and non-parietal lesions. Cortex, 40, 379-390.

Rainville, C., Giroire, J. M., Periot, M., Cuny, E., \& Mazaux, J. M. (2003). The impact of right subcortical lesions on executive functions and spatio-cognitive abilities: A case study. Neurocase, 9(4), 356-367.

Rode, G., Thomas-Antérion, C., Luauté, J., Jacquin-Courtois, S., Ciancia, S., Rossetti, Y. et al. (2005). Évaluation des incapacités et de la qualité de vie des patients présentant des troubles cognitifs. Annales de réadaptation et de médicine physique, 48, 376-391.

Shamay-Tsoory, S. G., Tomer, R., Berger, B. D., \& Aharon-Peretz, J. (2003). Characterization of empathy deficits following prefrontal brain damage: The role of the right ventromedial prefrontal cortex. Journal of Cognitive Neuroscience, 15(3), 324-337.

Siksou, M. (2005). Introduction à la neuropsychologie. Paris: Dunod.

Snyder, P. J., Nussbaum, P. D., \& Robins, D. L. (2006). Clinical neuropsychology. Washington: APA.

Spinazzola, L., Cubelli, R., \& Della Sala, S. (2003). Impairments of trunk movements following left or right hemisphere lesions: Dissociation between apraxic errors and postural instability. Brain, 126(12), 2656-2666.

Springer, S. P., \& Deutsch, G. (1998). Cérebro esquerdo, cérebro direito. São Paulo: Summus.

Tompkins, C. A, Fassbinder, W., Lehman-Blake, M. T., \& Baumgaertner, A. (2002). The nature and implications of right hemisphere language disorders: Issues in search of answer. In A. E. Hillis (Ed.), The handbook of adult language disorders - integrating cognitive neuropsychology, neurology, and rehabilitation (pp. 429-448). New York: Psychology Press.

Venegas, V., Manríquez, M., Prieto, H., Morales, A. M., Caballero, S., \& Rona, E. (1998). Síndrome del hemisfério derecho em niños: uma entidad clínica a definir. Revista Chilena de Neuro-Psiquiatría, 36, 108-113. 
Verfaellie, M., \& Heilman, K. M. (2006). Neglect syndromes. In P. J. Snyder, P. D. Nussbaum \& D. L. Robins (Eds.), Clinical neuropsychology (pp. 489-507). Washington: APA.

Wildgruber, D., Riecker, A., Hertrich, I., Erb, M., Grodd, W, Ethofer, T. et al. (2005). Identification of emotional intonation evaluated by fMRI. Neuroimage, 24, 1233-1241.

Wilkinson, D., Ko, P., Kilduff, P., McGlinchey, R., \& Milberg, W. (2005). Improvement of a face perception deficit via subsensory galvanic vestibular stimulation. Journal of the International Neuropsychological Society, 11(7), 925-929.

Recebido em: 17/09/2006

Aceito em: 28/10/2006 